PROCEEDINGS OF THE

AMERICAN MATHEMATICAL SOCIETY

Volume 137, Number 12, December 2009, Pages 4157-4167

S 0002-9939(09)10008-4

Article electronically published on July 14, 2009

\title{
NONCOMMUTATIVE $L_{p}$-SPACE AND OPERATOR SYSTEM
}

\author{
KYUNG HOON HAN
}

(Communicated by Marius Junge)

\begin{abstract}
We show that noncommutative $L_{p}$-spaces satisfy the axioms of the (nonunital) operator system with a dominating constant $2^{\frac{1}{p}}$. Therefore, noncommutative $L_{p}$-spaces can be embedded into $B(H) 2^{\frac{1}{p}}$-completely isomorphically and complete order isomorphically.
\end{abstract}

\section{INTRODUCTION}

A unital involutive subspace of $B(H)$ had been abstractly characterized by Choi and Effros $\mathrm{CE}$. Their axioms are based on observations of the relationship between the unit, the matrix order, and the matrix norm of the unital involutive subspace of $B(H)$. Indeed, the unit and the matrix order can be used to determine the matrix norm by applying

$$
\|x\|=\inf \left\{\lambda>0:-\lambda I \leq\left(\begin{array}{cc}
0 & x \\
x^{*} & 0
\end{array}\right) \leq \lambda I\right\}
$$

for a unital involutive subspace $X$ of $B(H)$ and $x \in M_{n}(X)$.

The abstract characterization of a nonunital involutive subspace of $B(H)$ was completed by Werner [W]. The axioms are based on observations of the relationship between the matrix norm and the matrix order of the involutive subspace of $B(H)$. Since the unit may be absent, the above equality is replaced by

$$
\|x\|=\sup \left\{\left|\varphi\left(\left(\begin{array}{cc}
0 & x \\
x^{*} & 0
\end{array}\right)\right)\right|: \varphi \in M_{2 n}(X)_{1,+}^{*}\right\}
$$

for an involutive subspace $X$ of $B(H)$ and $x \in M_{n}(X)$. We denote by $M_{2 n}(X)_{1,+}^{*}$ the set of positive contractive functionals on $M_{2 n}(X)$.

A complex involutive vector space $X$ is called a matrix ordered vector space if for each $n \in \mathbb{N}$, there is a set $M_{n}(X)_{+} \subset M_{n}(X)_{s a}$ such that

(1) $M_{n}(X)_{+} \cap\left[-M_{n}(X)_{+}\right]=\{0\}$ for all $n \in \mathbb{N}$,

(2) $M_{n}(X)_{+} \oplus M_{m}(X)_{+} \subset M_{n+m}(X)_{+}$for all $m, n \in \mathbb{N}$,

(3) $\gamma^{*} M_{m}(X)_{+} \gamma \subset M_{n}(X)_{+}$for each $m, n \in \mathbb{N}$ and all $\gamma \in M_{m ., n}(\mathbb{C})$.

One might infer from these conditions that $M_{n}(X)_{+}$is actually a cone.

An operator space $X$ is called a matrix ordered operator space iff $X$ is a matrix ordered vector space and for every $n \in \mathbb{N}$,

Received by the editors July 13, 2008, and, in revised form, February 16, 2009, and March 20, 2009 .

2000 Mathematics Subject Classification. Primary 46L07, 46L52, 47L07.

This work was supported by the BK21 project of the Ministry of Education, Korea.

(C)2009 American Mathematical Society Reverts to public domain 28 years from publication 
(1) the *-operation is an isometry on $M_{n}(X)$ and

(2) the cones $M_{n}(X)_{+}$are closed.

Suppose $X$ is a matrix ordered operator space. For $x \in M_{n}(X)$, the modified numerical radius is defined by

$$
\nu_{X}(x)=\sup \left\{\left|\varphi\left(\left(\begin{array}{cc}
0 & x \\
x^{*} & 0
\end{array}\right)\right)\right|: \varphi \in M_{2 n}(X)_{1,+}^{*}\right\} .
$$

We call a matrix ordered operator space an operator system iff there is a $k>0$ such that for all $n \in \mathbb{N}$ and $x \in M_{n}(x)$,

$$
\|x\| \leq k \nu_{X}(x) .
$$

Since $\nu_{X}(x) \leq\|x\|$ always holds, we can say that an operator system is a matrix ordered operator space such that the operator space norm and the modified numerical radius are equivalent uniformly for all $n \in \mathbb{N}$.

Werner showed that $X$ is an operator system if and only if there is a complete order isomorphism $\Phi$ from $X$ onto an involutive subspace of $B(H)$, which is a complete topological onto-isomorphism [W]. Hence, the operator system is an abstract characterization of the involutive subspace of $B(H)$ in the completely isomorphic and complete order isomorphic sense.

In this paper, noncommutative $L_{p}$-space is meant in the sense of Haagerup, which is based on Tomita-Takesaki theory Te1. While the noncommutative $L_{p^{-}}$ spaces arising from semifinite von Neumann algebras are a natural generalization of classical $L_{p}$-spaces $\left[\mathrm{S}\right.$, Ta], noncommutative $L_{p}$-spaces arising from type III von Neumann algebras are quite complicated. Recently, the reduction method approximating a general noncommutative $L_{p}$-space by tracial noncommutative $L_{p}$-spaces has been developed [HJX].

Noncommutative $L_{p}$-spaces can also be obtained by the complex interpolation method. There exist a canonical matrix order and a canonical operator space structure on each noncommutative $L_{p}$-space. The matrix order is given by the positive cones $L_{p}\left(M_{n}(\mathcal{M})\right)^{+}$, and the operator space structure is given by the complex interpolation

$$
M_{n}\left(L_{p}(\mathcal{M})\right)=\left(M_{n}(\mathcal{M}), M_{n}\left(\mathcal{M}_{*}^{o p}\right)\right)_{\frac{1}{p}} .
$$

We refer to [K, Pi1, Te2] for the details, and the reference [JRX, Section 4] is recommended for the summary. In particular, its discrete noncommutative vector valued $L_{p}$-space $S_{p}^{n}\left(L_{p}(\mathcal{M})\right)$ is given by

$$
S_{p}^{n}\left(L_{p}(\mathcal{M})\right)=L_{p}\left(M_{n}(\mathcal{M})\right) .
$$

We refer to $\mathrm{Pi2}$ for general information on noncommutative vector valued $L_{p^{-}}$ spaces.

The purpose of this paper is to show that noncommutative $L_{p}$-spaces satisfy the axioms of the operator system with a dominating constant $2^{\frac{1}{p}}$. As a corollary, we obtain the following embedding theorem: noncommutative $L_{p}$-spaces can be embedded into $B(H) 2^{\frac{1}{p}}$-completely isomorphically and complete order isomorphically. 


\section{Noncommutative $L_{p}$-SPACE And operator System}

If $X$ is a matrix ordered operator space, we regard $S_{p}^{n}(X)$ as an operator space having the same matrix order structure with $M_{n}(X)$. That is, we do not distinguish between $S_{p}^{n}(X)$ and $M_{n}(X)$ as matrix ordered vector spaces.

For a noncommutative $L_{p}$-space $L_{p}(\mathcal{M})$, it is more efficient to describe $S_{p}^{n}\left(L_{p}(\mathcal{M})\right)$ rather than $M_{n}\left(L_{p}(\mathcal{M})\right)$. So, we change the modified numerical radius into a form more adequate to noncommutative $L_{p}$-spaces. For a matrix ordered operator space $X$ and $x \in M_{n}(X)$, we define $\nu_{X}^{p}(x)$ as

$$
\nu_{X}^{p}(x)=2^{-\frac{1}{p}} \sup \left\{\left|\varphi\left(\left(\begin{array}{cc}
0 & x \\
x^{*} & 0
\end{array}\right)\right)\right|: \varphi \in S_{p}^{2 n}(X)_{1,+}^{*}\right\}
$$

where $S_{p}^{2 n}(X)_{1,+}^{*}$ denotes the set of positive contractive functionals on $S_{p}^{2 n}(X)$. Note that a functional $\varphi$ is positive on $S_{p}^{2 n}(X)$ if and only if it is positive on $M_{2 n}(X)$ because $S_{p}^{2 n}(X)$ and $M_{2 n}(X)$ have the same order structure. According to the following lemma, the uniform equivalence of a matrix norm and $\nu_{X}$ can be confirmed by showing the uniform equivalence of the $S_{p}^{n}$-norm and $\nu_{X}^{p}$.

Lemma 2.1. Suppose $X$ is a matrix ordered operator space and there is a constant $k>0$ satisfying $\|x\|_{S_{p}^{n}(X)} \leq k \nu_{X}^{p}(x)$ for any $x \in M_{n}(X)$. Then we have

$$
\|x\|_{M_{n}(X)} \leq k \nu_{X}(x)
$$

for any $x \in M_{n}(X)$. Hence, $X$ is an operator system.

Proof. For $a, b \in\left(S_{2 p}^{n}\right)_{1}$, we have

$$
\left\|\left(\begin{array}{cc}
a & 0 \\
0 & b^{*}
\end{array}\right)\right\|_{S_{2 p}^{2 n}}=\left(\|a\|_{S_{2 p}^{n}}^{2 p}+\left\|b^{*}\right\|_{S_{2 p}^{n}}^{2 p}\right)^{\frac{1}{2 p}} \leq 2^{\frac{1}{2 p}} .
$$

From [Pi2, Theorem 1.5], the map

$$
\left(\begin{array}{ll}
x_{11} & x_{12} \\
x_{21} & x_{22}
\end{array}\right) \mapsto 2^{-\frac{1}{p}} \varphi\left(\left(\begin{array}{cc}
a & 0 \\
0 & b^{*}
\end{array}\right)\left(\begin{array}{ll}
x_{11} & x_{12} \\
x_{21} & x_{22}
\end{array}\right)\left(\begin{array}{cc}
a^{*} & 0 \\
0 & b
\end{array}\right)\right)
$$

for $\varphi \in S_{p}^{2 n}(X)_{1,+}^{*}$ defines a positive contractive functional on $M_{2 n}(X)$. It follows that

$$
\begin{aligned}
\nu_{X}(x) & =\sup \left\{\mid \varphi\left(\left(\begin{array}{cc}
0 & x \\
x^{*} & 0
\end{array}\right) \mid: \varphi \in M_{2 n}(X)_{1,+}^{*}\right\}\right. \\
& \geq 2^{-\frac{1}{p}} \sup \left\{\left|\varphi\left(\left(\begin{array}{cc}
a & 0 \\
0 & b^{*}
\end{array}\right)\left(\begin{array}{cc}
0 & x \\
x^{*} & 0
\end{array}\right)\left(\begin{array}{cc}
a^{*} & 0 \\
0 & b
\end{array}\right)\right)\right|: \varphi \in S_{p}^{2 n}(X)_{1,+}^{*}, a, b \in\left(S_{2 p}^{n}\right)_{1}\right\} \\
& =2^{-\frac{1}{p}} \sup \left\{\mid \varphi\left(\left(\begin{array}{cc}
0 & a x b \\
b^{*} x^{*} a^{*} & 0
\end{array}\right) \mid: \varphi \in S_{p}^{2 n}(X)_{1,+}^{*}, a, b \in\left(S_{2 p}^{n}\right)_{1}\right\}\right. \\
& =\sup \left\{\nu_{X}^{p}(a x b): a, b \in\left(S_{2 p}^{n}\right)_{1}\right\} \\
& \geq k^{-1} \sup \left\{\|a x b\|_{S_{p}^{n}(X)}: a, b \in\left(S_{2 p}^{n}\right)_{1}\right\} \\
& =k^{-1}\|x\|_{M_{n}(X)}
\end{aligned}
$$

for $x \in M_{n}(X)$. For the last equality, see [Pi2, Lemma 1.7].

Lemma 2.2. Suppose $a$ is a bounded linear operator on a Hilbert space and $a=v|a|$ is its polar decomposition. Let $f$ be a continuous function defined on $[0, \infty)$ with 


$$
\begin{array}{r}
f(0)=0 . \text { Then }\left(\begin{array}{cc}
\left|a^{*}\right| & a \\
a^{*} & |a|
\end{array}\right) \text { is a positive operator and the equality } \\
\qquad f\left(\frac{1}{2}\left(\begin{array}{cc}
\left|a^{*}\right| & a \\
a^{*} & |a|
\end{array}\right)\right)=\frac{1}{2}\left(\begin{array}{cc}
f\left(\left|a^{*}\right|\right) & f\left(\left|a^{*}\right|\right) v \\
v^{*} f\left(\left|a^{*}\right|\right) & f(|a|)
\end{array}\right)
\end{array}
$$

holds.

Proof. The positivity follows from $[\mathrm{Pa}$, Exercise 8.8 (vi)]. By using the Weierstrass theorem and the right polar decomposition $a=\left|a^{*}\right| v$, it is sufficient to show that the equality

$$
\left(\begin{array}{cc}
\left|a^{*}\right| & a \\
a^{*} & |a|
\end{array}\right)^{n}=2^{n-1}\left(\begin{array}{cc}
\left|a^{*}\right|^{n} & \left|a^{*}\right|^{n-1} a \\
a^{*}\left|a^{*}\right|^{n-1} & |a|^{n}
\end{array}\right)
$$

holds for $n \geq 1$. We proceed by mathematical induction. Since $a^{*}\left(a a^{*}\right)^{k} a=$ $\left(a^{*} a\right)^{k+1}$ for $k \geq 0$, we have

$$
a^{*}\left|a^{*}\right|^{n-1} a=\left(a^{*} a\right)^{\frac{n-1}{2}+1}=|a|^{n+1} .
$$

Similarly, $a\left(a^{*} a\right)^{k}=\left(a a^{*}\right)^{k} a$ implies

$$
a|a|^{n}=\left|a^{*}\right|^{n} a .
$$

It follows that

$$
\begin{aligned}
& \left(\begin{array}{cc}
\left|a^{*}\right| & a \\
a^{*} & |a|
\end{array}\right) \cdot 2^{n-1}\left(\begin{array}{cc}
\left|a^{*}\right|^{n} & \left|a^{*}\right|^{n-1} a \\
a^{*}\left|a^{*}\right|^{n-1} & |a|^{n}
\end{array}\right) \\
& =2^{n-1}\left(\begin{array}{cc}
\left|a^{*}\right|^{n+1}+\left|a^{*}\right|^{n+1} & \left|a^{*}\right|^{n} a+a|a|^{n} \\
a^{*}\left|a^{*}\right|^{n}+|a| a^{*}\left|a^{*}\right|^{n-1} & a^{*}\left|a^{*}\right|^{n-1} a+|a|^{n+1}
\end{array}\right) \\
& =2^{n}\left(\begin{array}{ll}
\left|a^{*}\right|^{n+1} & \left|a^{*}\right|^{n} a \\
a^{*}\left|a^{*}\right|^{n} & |a|^{n+1}
\end{array}\right) .
\end{aligned}
$$

In order to prove that the Schatten $p$-class $S_{p}$ is an operator system, Lemma 2.2 is sufficient. However, in order to prove that a general noncommutative $L_{p}$-space is an operator system, an unbounded version of Lemma 2.2 is needed.

We denote the Dirac measure massed at the zero point by $\delta_{0}$.

Lemma 2.3. Let a be a closed densely defined operator on a Hilbert space. Suppose $a=v|a|$ is a polar decomposition and $|a|=\int_{0}^{\infty} t d E$ is a spectral decomposition. Then

$$
\widetilde{E}=\frac{1}{2}\left(\begin{array}{cc}
v E v^{*} & v E \\
E v^{*} & v^{*} v E
\end{array}\right)+\delta_{0}\left(\begin{array}{cc}
I-\frac{1}{2} v v^{*} & -\frac{1}{2} v \\
-\frac{1}{2} v^{*} & I-\frac{1}{2} v^{*} v
\end{array}\right)
$$

is a spectral measure corresponding to $\frac{1}{2}\left(\begin{array}{cc}\left|a^{*}\right| & a \\ a^{*} & |a|\end{array}\right)$.

Proof. Let

$$
E^{\prime}=\frac{1}{2}\left(\begin{array}{cc}
v E v^{*} & v E \\
E v^{*} & v^{*} v E
\end{array}\right)
$$


Since $v^{*} v=E((0, \infty)), v^{*} v$ commutes with $E(\Delta)$ for any Borel set $\Delta$ in $[0, \infty)$. For all Borel sets $\Delta_{1}$ and $\Delta_{2}$ in $[0, \infty)$, we have

$$
\begin{aligned}
& E^{\prime}\left(\Delta_{1}\right) E^{\prime}\left(\Delta_{2}\right) \\
= & \frac{1}{4}\left(\begin{array}{cc}
v E\left(\Delta_{1}\right) v^{*} & v E\left(\Delta_{1}\right) \\
E\left(\Delta_{1}\right) v^{*} & v^{*} v E\left(\Delta_{1}\right)
\end{array}\right)\left(\begin{array}{cc}
v E\left(\Delta_{2}\right) v^{*} & v E\left(\Delta_{2}\right) \\
E\left(\Delta_{2}\right) v^{*} & v^{*} v E\left(\Delta_{2}\right)
\end{array}\right) \\
= & \frac{1}{4}\left(\begin{array}{cc}
v E\left(\Delta_{1}\right) v^{*} v E\left(\Delta_{2}\right) v^{*}+v E\left(\Delta_{1}\right) E\left(\Delta_{2}\right) v^{*} & v E\left(\Delta_{1}\right) v^{*} v E\left(\Delta_{2}\right)+v E\left(\Delta_{1}\right) v^{*}+v^{*} v E\left(\Delta_{1}\right) E\left(\Delta_{2}\right) v^{*} \\
E\left(\Delta_{1}\right) v^{*} v E\left(\Delta_{2}\right)+v^{*} v E\left(\Delta_{1}\right) v^{*} v E\left(\Delta_{2}\right)
\end{array}\right) \\
= & \frac{1}{2}\left(\begin{array}{cc}
v E\left(\Delta_{1} \cap \Delta_{2}\right) v^{*} & v E\left(\Delta_{1} \cap \Delta_{2}\right) \\
E\left(\Delta_{1} \cap \Delta_{2}\right) v^{*} & v^{*} v E\left(\Delta_{1} \cap \Delta_{2}\right)
\end{array}\right) \\
= & E^{\prime}\left(\Delta_{1} \cap \Delta_{2}\right) .
\end{aligned}
$$

Taking $\Delta_{1}=\Delta_{2}$, we see that $E^{\prime}$ is projection valued. The countable additivity with respect to the strong operator topology is obvious. Since $E(\{0\})=I-v^{*} v$, we have

$$
E^{\prime}(\{0\})=0
$$

From these, it is easy to check that $\widetilde{E}$ satisfies all the conditions of a spectral measure.

We still need to show that

$$
\frac{1}{2}\left\langle\left(\begin{array}{cc}
\left|a^{*}\right| & a \\
a^{*} & |a|
\end{array}\right)\left(\begin{array}{l}
h \\
k
\end{array}\right) \mid\left(\begin{array}{l}
h \\
k
\end{array}\right)\right\rangle=\int_{0}^{\infty} t d \widetilde{E}_{\xi, \xi}
$$

for $\xi=\left(\begin{array}{l}h \\ k\end{array}\right)$ and $h \in D\left(a^{*}\right), k \in D(a)$. For the left-hand side, we compute

$$
\begin{aligned}
\left\langle\left(\begin{array}{cc}
\left|a^{*}\right| & a \\
a^{*} & |a|
\end{array}\right)\left(\begin{array}{c}
h \\
k
\end{array}\right) \mid\left(\begin{array}{l}
h \\
k
\end{array}\right)\right\rangle & =\left\langle\left|a^{*}\right| h \mid h\right\rangle+\langle a k \mid h\rangle+\left\langle a^{*} h \mid k\right\rangle+\langle|a| k \mid k\rangle \\
& =\left\langle v|a| v^{*} h \mid h\right\rangle+\langle v|a| k \mid h\rangle+\left\langle|a| v^{*} h \mid k\right\rangle+\left\langle v^{*} v|a| k \mid k\right\rangle \\
= & \int_{0}^{\infty} t d E_{v^{*} h, v^{*} h}+\int_{0}^{\infty} t d E_{k, v^{*} h} \\
& +\int_{0}^{\infty} t d E_{v^{*} h, k}+\int_{0}^{\infty} t d E_{k, v^{*} v k .}
\end{aligned}
$$

For the right-hand side, we compute

$$
\begin{aligned}
E_{\xi, \xi}^{\prime}(\Delta) & =\frac{1}{2}\left\langle\left(\begin{array}{cc}
v E(\Delta) v^{*} & v E(\Delta) \\
E(\Delta) v^{*} & v^{*} v E(\Delta)
\end{array}\right)\left(\begin{array}{l}
h \\
k
\end{array}\right) \mid\left(\begin{array}{l}
h \\
k
\end{array}\right)\right\rangle \\
& =\frac{1}{2}\left(\left\langle v E(\Delta) v^{*} h \mid h\right\rangle+\langle v E(\Delta) k \mid h\rangle+\left\langle E(\Delta) v^{*} h \mid k\right\rangle+\left\langle v^{*} v E(\Delta) k \mid k\right\rangle\right) \\
& =\frac{1}{2}\left(E_{v^{*} h, v^{*} h}(\Delta)+E_{k, v^{*} h}(\Delta)+E_{v^{*} h, k}(\Delta)+E_{k, v^{*} v k}(\Delta)\right) .
\end{aligned}
$$

Lemma 2.4. Suppose $\mathcal{M}$ is a semifinite von Neumann algebra with a faithful semifinite normal trace $\tau$ and $a$ is a $\tau$-measurable operator with its polar decomposition $a=v|a|$. Let $f$ be a continuous function defined on $[0, \infty)$ with $f(0)=0$. 
Then $\left(\begin{array}{cc}\left|a^{*}\right| & a \\ a^{*} & |a|\end{array}\right)$ is a positive self-adjoint $\tau_{2}$-measurable operator and the equality

$$
f\left(\frac{1}{2}\left(\begin{array}{cc}
\left|a^{*}\right| & a \\
a^{*} & |a|
\end{array}\right)\right)=\frac{1}{2}\left(\begin{array}{cc}
f\left(\left|a^{*}\right|\right) & f\left(\left|a^{*}\right|\right) v \\
v^{*} f\left(\left|a^{*}\right|\right) & f(|a|)
\end{array}\right)
$$

holds.

Proof. Because we have

$$
\begin{aligned}
\tau_{2}(\tilde{E}((n, \infty)) & =\tau\left(\frac{1}{2} v E((n, \infty)) v^{*}\right)+\tau\left(\frac{1}{2} v^{*} v E(n, \infty)\right) \\
& =\tau(E(n, \infty)) \rightarrow 0
\end{aligned}
$$

$\left(\begin{array}{cc}\left|a^{*}\right| & a \\ a^{*} & |a|\end{array}\right)$ is $\tau_{2}$-measurable.

Suppose $E$ is a spectral measure corresponding to $|a|$. We let

$$
a_{n}=a E([0, n]) .
$$

Then we have

$$
\left|a_{n}\right|=\left(E \left([0, n] a^{*} a E([0, n])^{\frac{1}{2}}=|a| E([0, n]) .\right.\right.
$$

Since $a_{n} v^{*}=v|a| E([0, n]) v^{*} \geq 0$ and $\left(a_{n} v^{*}\right)^{2}=a E([0, n]) v^{*} v E([0, n]) a^{*}=$ $a E([0, n]) a^{*}$, we have

$$
a_{n} v^{*}=\left(a E([0, n]) a^{*}\right)^{\frac{1}{2}}=\left|a_{n}^{*}\right|
$$

We compute

$$
\begin{aligned}
& \frac{1}{2}\left(\begin{array}{cc}
\left|a^{*}\right| & a \\
a^{*} & |a|
\end{array}\right) \widetilde{E}([0, n]) \\
& \quad=\frac{1}{4}\left(\begin{array}{cc}
\left|a^{*}\right| & a \\
a^{*} & |a|
\end{array}\right)\left(\begin{array}{cc}
v E([0, n]) v^{*} & v E([0, n]) \\
E([0, n]) v^{*} & v^{*} v E([0, n])
\end{array}\right) \\
& \quad=\frac{1}{4}\left(\begin{array}{cc}
\left|a^{*}\right| v E([0, n]) v^{*}+a E([0, n]) v^{*} & \left|a^{*}\right| v E([0, n])+a v^{*} v E([0, n]) \\
a^{*} v E([0, n]) v^{*}+|a| E([0, n]) v^{*} & a^{*} v E([0, n])+|a| v^{*} v E([0, n])
\end{array}\right) \\
& \quad=\frac{1}{2}\left(\begin{array}{cc}
\left|a_{n}^{*}\right| & a_{n} \\
a_{n}^{*} & \left|a_{n}\right|
\end{array}\right) .
\end{aligned}
$$

Since $\left|a_{n}\right|$ is bounded, we can apply Lemma 2.2. The partial isometry in the polar decomposition of $a_{n}$ is $v_{n}:=v E([0, n])$. It follows that

$$
\begin{aligned}
f\left(\frac{1}{2}\left(\begin{array}{cc}
\left|a^{*}\right| & a \\
a^{*} & |a|
\end{array}\right)\right) \widetilde{E}([0, n]) & =f\left(\frac{1}{2}\left(\begin{array}{cc}
\left|a_{n}^{*}\right| & a_{n} \\
a_{n}^{*} & \left|a_{n}\right|
\end{array}\right)\right) \\
& =\frac{1}{2}\left(\begin{array}{cc}
f\left(\left|a_{n}^{*}\right|\right) & f\left(\left|a_{n}^{*}\right|\right) v_{n} \\
v_{n}^{*} f\left(\left|a_{n}^{*}\right|\right) & f\left(\left|a_{n}\right|\right)
\end{array}\right) .
\end{aligned}
$$

Since $v^{*}\left|a_{n}^{*}\right|^{k} v=v^{*}\left(a_{n} v^{*}\right)^{k} v=\left|a_{n}\right|^{k}$ for any $k \geq 1$, we have

$$
v^{*} f\left(\left|a_{n}^{*}\right|\right) v=f\left(\left|a_{n}\right|\right) .
$$

Similarly, we also have

$$
v f\left(\left|a_{n}\right|\right) v^{*}=f\left(\left|a_{n}^{*}\right|\right) .
$$


The spectral measure for $\left|a^{*}\right|$ is $v E v^{*}+\delta_{0}\left(I-v v^{*}\right)$. It follows that

$$
\begin{aligned}
& \frac{1}{2}\left(\begin{array}{cc}
f\left(\left|a^{*}\right|\right) & f\left(\left|a^{*}\right|\right) v \\
v^{*} f\left(\left|a^{*}\right|\right) & f(|a|)
\end{array}\right) \widetilde{E}([0, n]) \\
= & \frac{1}{4}\left(\begin{array}{cc}
2 f\left(\left|a^{*}\right|\right) v E([0, n]) v^{*} & 2 f\left(\left|a^{*}\right|\right) v E([0, n]) \\
v^{*} f\left(\left|a^{*}\right|\right) v E([0, n]) v^{*}+f(|a|) E([0, n]) v^{*} & v^{*} f\left(\left|a^{*}\right|\right) v E([0, n])+f(|a|) v^{*} v E([0, n])
\end{array}\right) \\
= & \frac{1}{4}\left(\begin{array}{cc}
2 f\left(\left|a^{*}\right| v E([0, n]) v^{*}\right) & 2 f\left(\left|a^{*}\right| v E([0, n]) v^{*}\right) v_{n} \\
v_{n}^{*} f\left(\left|a^{*}\right| v E([0, n]) v^{*}\right)+v_{n}^{*} v f(|a| E([0, n])) v^{*} & v^{*} f\left(\left|a^{*}\right| v E([0, n]) v^{*}\right) v+f(|a| E([0, n]))
\end{array}\right) \\
= & \frac{1}{2}\left(\begin{array}{cc}
f\left(\left|a_{n}^{*}\right|\right) & f\left(\left|a_{n}^{*}\right|\right) v_{n} \\
v_{n}^{*} f\left(\left|a_{n}^{*}\right|\right) & f\left(\left|a_{n}\right|\right)
\end{array}\right) .
\end{aligned}
$$

Because the set $\bigcup_{n=1}^{\infty} \operatorname{ran} \widetilde{E}([0, n])$ is $\tau_{2}$-dense, we obtain the desired result from [Te1, Proposition I.12].

Lemma 2.5. Suppose $\mathcal{M}$ is a von Neumann algebra with a faithful semifinite normal weight $\varphi$. If $a$ is an element of Haagerup's noncommutative $L_{p}$-space $L_{p}(\mathcal{M})$, then we have

$$
\left(\begin{array}{cc}
\left|a^{*}\right| & a \\
a^{*} & |a|
\end{array}\right) \in L_{p}\left(M_{2}(\mathcal{M})\right)_{+} \quad \text { and } \quad\left\|\left(\begin{array}{cc}
\left|a^{*}\right| & a \\
a^{*} & |a|
\end{array}\right)\right\|_{L_{p}\left(M_{2}(\mathcal{M})\right)}=2\|a\|_{L_{p}(\mathcal{M})} .
$$

Proof. Let $\theta$ be the dual action of $\mathbb{R}$ on $\mathcal{M} \rtimes_{\sigma^{\varphi}} \mathbb{R}$. Then $i d_{M_{2}} \otimes \theta$ is the dual action of $\mathbb{R}$ on $M_{2}(\mathcal{M}) \rtimes_{\sigma^{t r \otimes \varphi}} \mathbb{R}$. Because

$$
\begin{aligned}
\left(i d_{M_{2}} \otimes \theta_{s}\right)\left(\begin{array}{cc}
\left|a^{*}\right| & a \\
a^{*} & |a|
\end{array}\right) & =\left(\begin{array}{cc}
\theta_{s}\left(\left|a^{*}\right|\right) & \theta_{s}(a) \\
\theta_{s}\left(a^{*}\right) & \theta_{s}(|a|)
\end{array}\right) \\
& =e^{-\frac{s}{p}}\left(\begin{array}{cc}
\left|a^{*}\right| & a \\
a^{*} & |a|
\end{array}\right),
\end{aligned}
$$

we have

$$
\left(\begin{array}{cc}
\left|a^{*}\right| & a \\
a^{*} & |a|
\end{array}\right) \in L_{p}\left(M_{2}(\mathcal{M})\right)_{+} .
$$

From Lemma 2.4, it follows that

$$
\begin{aligned}
\left\|\left(\begin{array}{cc}
\left|a^{*}\right| & a \\
a^{*} & |a|
\end{array}\right)\right\|_{L_{p}\left(M_{2}(\mathcal{M})\right)}^{p} & =\left\|\left(\begin{array}{cc}
\left|a^{*}\right| & a \\
a^{*} & |a|
\end{array}\right)^{p}\right\|_{L_{1}\left(M_{2}(\mathcal{M})\right)} \\
& =2^{p-1}\left\|\left(\begin{array}{cc}
\left|a^{*}\right|^{p} & \left|a^{*}\right|^{p} v \\
v^{*}\left|a^{*}\right|^{p} & |a|^{p}
\end{array}\right)\right\|_{L_{1}\left(M_{2}(\mathcal{M})\right)} \\
& =2^{p-1}\left(\left\|\left|a^{*}\right|^{p}\right\|_{L_{1}(\mathcal{M})}+\left\||a|^{p}\right\|_{L_{1}(\mathcal{M})}\right) \\
& =2^{p}\|a\|_{L_{p}(\mathcal{M})}^{p}
\end{aligned}
$$

Theorem 2.6. Suppose $\mathcal{M}$ is a von Neumann algebra with a faithful semifinite normal weight $\varphi$. Its noncommutative $L_{p}$-space $L_{p}(\mathcal{M})$ is an operator system with

$$
\|a\|_{M_{n}\left(L_{p}(\mathcal{M})\right)} \leq 2^{\frac{1}{p}} \nu_{L_{p}(\mathcal{M})}(a), \quad a \in M_{n}\left(L_{p}(\mathcal{M})\right) .
$$

Proof. Let $a \in L_{p}\left(M_{n}(\mathcal{M})\right)$ with its right polar decomposition $a=\left|a^{*}\right| v$. Because

$$
\begin{aligned}
\|a\|_{L_{p}\left(M_{n}(\mathcal{M})\right)} & =\left\|\left|a^{*}\right| v\right\|_{L_{p}\left(M_{n}(\mathcal{M})\right)} \\
& \leq\left\|a^{*}\right\|_{L_{p}\left(M_{n}(\mathcal{M})\right)},
\end{aligned}
$$

the involution is an isometry on $L^{p}\left(M_{n}(\mathcal{M})\right)$, which can be identified with $S_{p}^{n}\left(L^{p}(\mathcal{M})\right)$. From [Pi2, Lemma 1.7], the involution is also an isometry on $M_{n}\left(L^{p}(\mathcal{M})\right)$. The Hölder inequality and [Te1, Proposition II.33] show that the 
positive cone $L^{p}\left(M_{n}(\mathcal{M})\right)_{+}$is closed. Hence, the noncommutative $L_{p}$-space is a matrix ordered operator space.

Let $a \in S_{p}^{n}\left(L_{p}(\mathcal{M})\right)$ and $\frac{1}{p}+\frac{1}{q}=1$. From Lemma 2.5. it follows that

$$
\begin{aligned}
\|a\|_{L_{p}\left(M_{n}(\mathcal{M})\right)} & =\sup \left\{\left|\operatorname{tr}_{\mathcal{M}}\left(b^{*} a\right)\right|: b \in L_{q}\left(M_{n}(\mathcal{M})\right)_{1}\right\} \\
& =\sup \left\{\operatorname{Re}_{\left.\operatorname{tr}_{\mathcal{M}}\left(b^{*} a\right): b \in L_{q}\left(M_{n}(\mathcal{M})\right)_{1}\right\}}\right. \\
& =\sup \left\{\frac{1}{2}\left(\operatorname{tr}_{\mathcal{M}}\left(b^{*} a\right)+\operatorname{tr}_{\mathcal{M}}\left(b a^{*}\right)\right): b \in L_{q}\left(M_{n}(\mathcal{M})\right)_{1}\right\} \\
& =\sup \left\{\frac{1}{2} \operatorname{tr}_{M_{2}(\mathcal{M})}\left(\begin{array}{cc}
b a^{*} & \left|b^{*}\right| a \\
|b| a^{*} & b^{*} a
\end{array}\right): b \in L_{q}\left(M_{n}(\mathcal{M})\right)_{1}\right\} \\
& =\sup \left\{\frac{1}{2} \operatorname{tr}_{M_{2}(\mathcal{M})}\left(\left(\begin{array}{cc}
\left|b^{*}\right| & b \\
b^{*} & |b|
\end{array}\right)\left(\begin{array}{cc}
0 & a \\
a^{*} & 0
\end{array}\right)\right): b \in L_{q}\left(M_{n}(\mathcal{M})\right)_{1}\right\} \\
& \leq \sup \left\{\varphi\left(\left(\begin{array}{cc}
0 & a \\
a^{*} & 0
\end{array}\right)\right): \varphi \in L_{p}\left(M_{2 n}(\mathcal{M})\right)_{1,+}^{*}\right\} \\
& =2^{\frac{1}{p}} \nu_{L_{p}(\mathcal{M})}^{p}(a) .
\end{aligned}
$$

Thus, from Lemma 2.1, we obtain the desired result.

By combining this with [W, Theorem 4.15], we obtain the following embedding theorem.

Corollary 2.7. Noncommutative $L_{p}$-spaces can be embedded into $B(H) 2^{\frac{1}{p}}$ completely isomorphically and complete order isomorphically.

For a $C^{*}$-algebra $A$, we define an involution on its dual space $A^{*}$ as

$$
\varphi^{*}(a)=\varphi\left(a^{*}\right)^{*}, \quad \varphi \in A^{*}, a \in A .
$$

Along the line of operator space duality, we identify $M_{n}\left(A^{*}\right)$ and $M_{n}(A)^{*}$ algebraically by using the parallel duality pairing

$$
\left\langle\left[\varphi_{i j}\right],\left[a_{i j}\right]\right\rangle=\sum_{i, j} \varphi_{i j}\left(a_{i j}\right), \quad \varphi_{i j} \in A^{*}, a_{i j} \in A .
$$

We can now define the positive cone $M_{n}\left(A^{*}\right)_{+}$. However, the duality between the von Neumann algebra $M_{n}(\mathcal{M})$ and its noncommutative $L_{1}$-space $L^{1}\left(M_{n}(\mathcal{M})\right)$ is given by the trace duality pairing

$$
\left\langle\left[a_{i j}\right],\left[b_{i j}\right]\right\rangle=\operatorname{tr}_{M_{n}} \otimes \operatorname{tr}_{\mathcal{M}}\left(\left[a_{i j}\right]\left[b_{i j}\right]\right)=\sum_{i, j} \operatorname{tr}_{\mathcal{M}}\left(a_{i j} b_{j i}\right) .
$$

Hence, we must be more careful.

For an operator space $V$, its opposite operator space $V^{o p}$ is defined by

$$
\left\|\left[v_{i j}^{o p}\right]\right\|_{M_{n}\left(V^{o p}\right)}=\left\|\left[v_{j i}\right]\right\|_{M_{n}(V)} .
$$

Note that $V^{o p}$ and $V$ are the same normed spaces. For a matrix ordered operator space $X$, we define its opposite matrix ordered operator space $X^{o p}$ as the opposite operator space with the matrix order

$$
\left[x_{i j}^{o p}\right] \geq 0 \Leftrightarrow\left[x_{j i}\right] \geq 0 .
$$

A functional $\varphi: M_{n}(X) \rightarrow \mathbb{C}$ can be written as

$$
\varphi=\left[\varphi_{i j}\right], \quad \varphi\left(\left[x_{i j}\right]\right)=\sum_{i, j} \varphi_{i j}\left(x_{i j}\right) .
$$


Let $\tilde{\varphi}=\left[\varphi_{j i}\right]$. Then we have

$$
\varphi\left(\left[x_{i j}\right]\right)=\tilde{\varphi}\left(\left[x_{j i}\right]\right)
$$

A functional $\varphi: M_{n}(X) \rightarrow \mathbb{C}$ is positive and contractive if and only if $\tilde{\varphi}$ : $M_{n}\left(X^{o p}\right) \rightarrow \mathbb{C}$ is positive and contractive. From this, we see that $X$ is an operator system if and only if $X^{o p}$ is an operator system.

Corollary 2.8. The dual space of a $C^{*}$-algebra is an operator system.

Proof. The bidual $A^{* *}$ of a $C^{*}$-algebra $A$ can be identified with the enveloping von Neumann algebra of $A$. The opposite matrix ordered operator space $\left(A^{*}\right)^{o p}$ is completely isometric and completely order isomorphic to $L_{1}\left(A^{* *}\right)$ by [Te1, Theorem II.7]. Hence, the dual space $A^{*}$ is an operator system.

Finally, we show that the constant $2^{\frac{1}{p}}$ in the inequality

$$
\|a\|_{L_{p}\left(M_{n}(\mathcal{M})\right)} \leq 2^{\frac{1}{p}} \nu_{L_{p}(\mathcal{M})}^{p}(a)
$$

is the best one. However, this does not imply that $2^{\frac{1}{p}}$ is the best constant in Theorem 2.6. Unexpectedly, it is achieved by the 2-dimensional commutative case. We consider an element $(1,1)$ in $\ell_{p}^{2}$. It is sufficient to show that

$$
\nu_{\ell_{p}^{2}}^{p}((1,1)) \leq 1
$$

For $\frac{1}{p}+\frac{1}{q}=1$, we let

$$
\left(\begin{array}{cccc}
a_{1} & \cdot & b_{1} & \cdot \\
\cdot & a_{2} & \cdot & b_{2} \\
\bar{b}_{1} & \cdot & d_{1} & \cdot \\
\cdot & \bar{b}_{2} & \cdot & d_{2}
\end{array}\right) \in\left(S_{q}^{4}\right)_{1,+}
$$

We have

$$
\begin{aligned}
0 & \left.\leq \operatorname{tr}\left(\begin{array}{cccc}
a_{1} & \cdot & b_{1} & \cdot \\
\cdot & a_{2} & \cdot & b_{2} \\
\bar{b}_{1} & \cdot & d_{1} & \cdot \\
\cdot & \bar{b}_{2} & \cdot & d_{2}
\end{array}\right)\left(\begin{array}{cccc}
1 & \cdot & -1 & \cdot \\
\cdot & 1 & \cdot & -1 \\
-1 & \cdot & 1 & \cdot \\
\cdot & -1 & \cdot & 1
\end{array}\right)\right) \\
& =a_{1}+a_{2}+d_{1}+d_{2}-b_{1}-b_{2}-\bar{b}_{1}-\bar{b}_{2} .
\end{aligned}
$$


It follows that

$$
\begin{aligned}
&\left.\operatorname{tr}\left(\begin{array}{cccc}
a_{1} & \cdot & b_{1} & \cdot \\
\cdot & a_{2} & \cdot & b_{2} \\
\bar{b}_{1} & \cdot & d_{1} & \cdot \\
\cdot & \bar{b}_{2} & \cdot & d_{2}
\end{array}\right)\left(\begin{array}{cccc}
\cdot & \cdot & 1 & \cdot \\
\cdot & \cdot & \cdot & 1 \\
1 & \cdot & \cdot & \cdot \\
\cdot & 1 & \cdot & \cdot
\end{array}\right)\right)=b_{1}+b_{2}+\bar{b}_{1}+\bar{b}_{2} \\
& \leq \frac{1}{2}\left(a_{1}+a_{2}+d_{1}+d_{2}+b_{1}+b_{2}+\bar{b}_{1}+\bar{b}_{2}\right) \\
&\left.=\frac{1}{2} \operatorname{tr}\left(\begin{array}{cccc}
a_{1} & \cdot & b_{1} & \cdot \\
\cdot & a_{2} & \cdot & b_{2} \\
\bar{b}_{1} & \cdot & d_{1} & \cdot \\
\cdot & \bar{b}_{2} & \cdot & d_{2}
\end{array}\right)\left(\begin{array}{cccc}
1 & \cdot & 1 & \cdot \\
\cdot & 1 & \cdot & 1 \\
1 & \cdot & 1 & \cdot \\
\cdot & 1 & \cdot & 1
\end{array}\right)\right) \\
& \leq \frac{1}{2}\left\|\left(\begin{array}{cccc}
1 & \cdot & 1 & \cdot \\
\cdot & 1 & \cdot & 1 \\
1 & \cdot & 1 & \cdot \\
\cdot & 1 & \cdot & 1
\end{array}\right)\right\|_{S_{p}^{4}} \\
& \\
&=\frac{1}{2}\left\|\left(\begin{array}{cccc}
1 & 1 & \cdot & \cdot \\
1 & 1 & \cdot & \cdot \\
\cdot & \cdot & 1 & 1 \\
\cdot & \cdot & 1 & 1
\end{array}\right)\right\|_{S_{p}^{4}} \\
&=2^{\frac{1}{p}} .
\end{aligned}
$$

In the same manner, we can also show that

$$
\operatorname{tr}\left(\left(\begin{array}{cccc}
a_{1} & \cdot & b_{1} & \cdot \\
\cdot & a_{2} & \cdot & b_{2} \\
\bar{b}_{1} & \cdot & d_{1} & \cdot \\
\cdot & \bar{b}_{2} & \cdot & d_{2}
\end{array}\right)\left(\begin{array}{cccc}
\cdot & \cdot & 1 & \cdot \\
\cdot & \cdot & \cdot & 1 \\
1 & \cdot & \cdot & \cdot \\
\cdot & 1 & \cdot & \cdot
\end{array}\right)\right) \geq-2^{\frac{1}{p}}
$$

We obtain the inequality

$$
\nu_{\ell_{p}^{2}}^{p}((1,1)) \leq 1
$$

\section{REFERENCES}

[CE] M.-D. Choi and E. Effros, Injectivity and operator spaces, J. Funct. Anal. 24 (1997), 156-209. MR0430809 (55:3814)

[HJX] U. Haagerup M. Junge, and Q. Xu, A reduction method for noncommutative $L_{p}$-spaces and applications, Trans. Amer. Math. Soc., to appear.

[JRX] M. Junge, Z-J. Ruan and Q. Xu, Rigid $\mathcal{O} \mathcal{L}_{p}$ structures of non-commutative $L_{p}$-spaces associated with hyperfinite von Neumann algebras, Math. Scand. 96 (2005), 63-95. MR2142873 (2006b:46086)

[K] H. Kosaki, Applications of the complex interpolation method to a von Neumann algebra: Non-commutative $L^{p}$-spaces, J. Funct. Anal. 56 (1984), 29-78 MR735704 (86a:46085)

$[\mathrm{Pa}]$ V. I. Paulsen, Completely Bounded Maps and Operator Algebras, Cambridge Studies in Advanced Mathematics, 78, Cambridge University Press, Cambridge, UK, 2002. MR1976867 (2004c:46118)

[Pi1] G. Pisier, The operator Hilbert space OH, complex interpolation and tensor norms, Mem. Amer. Math. Soc. 122, No. 585, 1996. MR1342022 (97a:46024)

[Pi2] G. Pisier, Non-commutative vector valued $L_{p}$-spaces and completely p-summing maps, Astérisque 247, 1998. MR1648908 (2000a:46108)

[S] I. E. Segal, A non-commutative extension of abstract integration, Ann. of Math. (2) 57 (1953), 401-457. MR0054864(14:991f)

[Ta] M. Takesaki, Theory of Operator Algebras. II, Encyclopaedia of Mathematical Sciences, Vol. 125, Springer-Verlag, 2002. MR1873025 (2002m:46083) 
[Te1] M. Terp, $L^{p}$ spaces associated with von Neumann algebras, Math. Institute, Copenhagen University, 1981.

[Te2] M. Terp, Interpolation spaces between a von Neumann algebra and its predual, J. Operator Theory 8 (1982), 327-360. MR677418 (85b:46075)

[W] W. Werner, Subspaces of $L(H)$ that are *-invariant, J. Funct. Anal. 193 (2002), 207-223. MR.1929500 (2003h:46086)

Department of Mathematical Sciences, Seoul National University, San 56-1 ShinRimDong, KwanAk-Gu, Seoul 151-747, Korea

E-mail address: kyunghoon.han@gmail.com 